\title{
The HOSTS survey for exo-zodiacal dust: preliminary results and future prospects
}

S. Ertel, G. M. Kennedy, D. Defrère, P. Hinz, A. B. Shannon, et al.

S. Ertel, G. M. Kennedy, D. Defrère, P. Hinz, A. B. Shannon, B. Mennesson, W. C. Danchi, C. Gelino, J. M. Hill, W. F. Hoffman, G. Rieke, E. Spalding, J. M. Stone, A. Vaz, A. J. Weinberger, P. Willems, O. Absil, P. Arbo, V. P. Bailey, C. Beichman, G. Bryden, E. C. Downey, O. Durney, S. Esposito, A. Gaspar, P. Grenz, C. A. Haniff, J. M. Leisenring, L. Marion, T. J. McMahon, R. Millan-Gabet, M. Montoya, K. M. Morzinski, E. Pinna, J. Power, A. Puglisi, A. Roberge, E. Serabyn, A. J. Skemer, K. Stapelfeldt, K. Y. L. Su, V. Vaitheeswaran, M. C. Wyatt, "The HOSTS survey for exo-zodiacal dust: preliminary results and future prospects," Proc. SPIE 10698, Space Telescopes and Instrumentation 2018: Optical, Infrared, and Millimeter Wave, 106981V (24 July 2018); doi: 10.1117/12.2313685

Event: SPIE Astronomical Telescopes + Instrumentation, 2018, Austin, Texas, United States 


\section{The HOSTS Survey for Exozodiacal Dust: Preliminary results and future prospects}

Ertel, S. ${ }^{a}$, Kennedy, G. M. ${ }^{\text {b,c }}$, Defrère, D. ${ }^{\mathrm{d}}$, Hinz, P. ${ }^{\mathrm{a}}$, Shannon, A. B. ${ }^{\mathrm{e}, \mathrm{f}}$, Mennesson, B. ${ }^{\mathrm{g}}$,

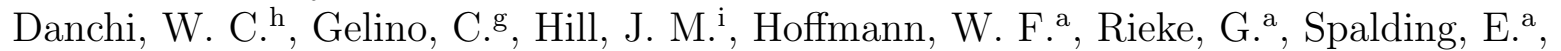
Stone, J. M.*a, Vaz, A. ${ }^{\text {a }}$, Weinberger, A. J. ${ }^{\mathrm{j}}$, Willems, P. ${ }^{\mathrm{g}}$, Absil, O. ${ }^{\mathrm{d}}$, Arbo, P. ${ }^{\mathrm{a}}$, Bailey, V. P. ${ }^{\mathrm{g}}$,

Beichman, C. ${ }^{\mathrm{k}}$, Bryden, G. ${ }^{\mathrm{g}}$, Downey, E. C. ${ }^{\mathrm{a}}$, Durney, O. ${ }^{\mathrm{a}}$, Esposito, S. ${ }^{\mathrm{l}}$, Gaspar, A. ${ }^{\mathrm{a}}$,

Grenz, P. ${ }^{a}$, Haniff, C. A. ${ }^{\mathrm{m}}$, Leisenring, J. M. ${ }^{\mathrm{a}}$, Marion, L. ${ }^{\mathrm{d}}$, McMahon, T. J. ${ }^{\mathrm{a}}$,

Millan-Gabet, R. ${ }^{\text {, }}$ Montoya, M. ${ }^{a}$, Morzinski, K. M. ${ }^{a}$, Pinna, E. ${ }^{1}$, Power, J. ${ }^{\text {, }}$ Puglisi, A. ${ }^{\text {, }}$ Roberge, A. ${ }^{\text {, }}$ Serabyn, E. ${ }^{g}$, Skemer, A. J. ${ }^{n}$, Stapelfeldt, K. ${ }^{g}$, Su, K. Y. L. ${ }^{a}$, Vaitheeswaran, V. ${ }^{\text {a }}$, and Wyatt, M. C. ${ }^{\circ}$

a Steward Observatory, Department of Astronomy, University of Arizona, 993 N. Cherry Ave, Tucson, AZ, 85721, USA

${ }^{\mathrm{b}}$ Department of Physics, University of Warwick, Gibbet Hill Road, Coventry CV4 7AL, UK

${ }^{\mathrm{c}}$ Centre for Exoplanets and Habitability, University of Warwick, Gibbet Hill Road, Coventry, CV4 7AL, UK

dSpace sciences, Technologies \& Astrophysics Research (STAR) Institute, University of Liège, Liège, Belgium

eDepartment of Astronomy and Astrophysics, The Pennsylvania State University, State College, PA 16801, USA

${ }^{\mathrm{f} C e n t e r ~ f o r ~ E x o p l a n e t s ~ a n d ~ H a b i t a b l e ~ W o r l d s, ~ T h e ~ P e n n s y l v a n i a ~ S t a t e ~ U n i v e r s i t y, ~ S t a t e ~}$ College, PA 16802, USA

${ }^{g}$ Jet Propulsion Laboratory, California Institute of Technology, 4800 Oak Grove Dr., Pasadena, CA 91109, USA

hNASA Goddard Space Flight Center, Exoplanets \& Stellar Astrophysics Laboratory, Code 667, Greenbelt, MD 20771, USA

${ }^{i}$ Large Binocular Telescope Observatory, University of Arizona, 933 N. Cherry Avenue, Tucson, AZ 85721, USA

jDepartment of Terrestrial Magnetism, Carnegie Institution of Washington, 5241 Broad

Branch Road NW, Washington, DC, 20015, USA

${ }^{k}$ NASA Exoplanet Science Institute, MS 100-22, California Institute of Technology, Pasadena, CA 91125, USA

${ }^{1}$ INAF-Osservatorio Astrofisico di Arcetri, Largo E. Fermi 5, I-50125 Firenze, Italy

${ }^{\mathrm{m}}$ Cavendish Laboratory, University of Cambridge, JJ Thomson Avenue, Cambridge CB3 0HE, UK

${ }^{\mathrm{n}}$ Astronomy Department, University of California Santa Cruz, 1156 High Street, Santa Cruz, CA 95064, USA

'Institute of Astronomy, University of Cambridge, Madingley Road, Cambridge CB3 0HA, UK

* Hubble fellow.

Send correspondence to Steve Ertel, E-mail: sertel@email.arizona.edu

Space Telescopes and Instrumentation 2018: Optical, Infrared, and Millimeter Wave, edited by Makenzie Lystrup, Howard A. MacEwen, Giovanni G. Fazio, Proc. of SPIE Vol. 10698, 106981V (C) 2018 SPIE · CCC code: $0277-786 X / 18 / \$ 18 \cdot$ doi: $10.1117 / 12.2313685$ 


\begin{abstract}
The presence of large amounts of dust in the habitable zones of nearby stars is a significant obstacle for future exo-Earth imaging missions. We executed the HOSTS (Hunt for Observable Signatures of Terrestrial Systems) survey to determine the typical amount of such exozodiacal dust around a sample of nearby main sequence stars. The majority of the data have been analyzed and we present here an update of our ongoing work. Nulling interferometry in $N$ band was used to suppress the bright stellar light and to detect faint, extended circumstellar dust emission. We present an overview of the latest results from our ongoing work. We find seven new $N$ band excesses in addition to the high confidence confirmation of three that were previously known. We find the first detections around Sun-like stars and around stars without previously known circumstellar dust. Our overall detection rate is $23 \%$. The inferred occurrence rate is comparable for early type and Sun-like stars, but decreases from $71_{-20}^{+11} \%$ for stars with previously detected mid- to far-infrared excess to $11_{-4}^{+9} \%$ for stars without such excess, confirming earlier results at high confidence. For completed observations on individual stars, our sensitivity is five to ten times better than previous results. Assuming a lognormal luminosity function of the dust, we find upper limits on the median dust level around all stars without previously known mid to far infrared excess of 11.5 zodis at $95 \%$ confidence level. The corresponding upper limit for Sun-like stars is 16 zodis. An LBTI vetted target list of Sun-like stars for exo-Earth imaging would have a corresponding limit of 7.5 zodis. We provide important new insights into the occurrence rate and typical levels of habitable zone dust around main sequence stars. Exploiting the full range of capabilities of the LBTI provides a critical opportunity for the detailed characterization of a sample of exozodiacal dust disks to understand the origin, distribution, and properties of the dust.
\end{abstract}

Keywords: Exo-zodiacal dust, Interferometry, High contrast imaging, Habitable zone, Exo-Earth imaging, Mid-infrared

\title{
1. INTRODUCTION
}

The presence of warm and hot dust in nearby planetary systems both provides important opportunities to study their architecture and dynamics and impairs our ability to directly image and characterize Earth-like planets. At temperatures between a few $100 \mathrm{~K}$ to $\sim 2000 \mathrm{~K}$, such exozodiacal dust clouds (exozodis for short) are located near the habitable zones (HZs, $[1,2,3]$ ) of their host stars and closer in $[4,5,6,7]$.

The dust may form from comet evaporation [8], local asteroid collisions (for young systems and at relatively large separations from the star, [9]), and collisions of large bodies further out in the system from where it migrates closer to the star due to Poynting-Robertson and stellar wind drag [10,11]. It gravitationally interacts with any planets in the system in addition to the star and is ground to (sub-)micron sizes through mutual collisions of dust grains, after which the stellar radiation and wind pressure cause the grains to be expelled from the system. The dust distribution may trace the location of the dust producing planetesimals or in case of cometary origin the magnitude of the cometary activity. Structures in the dust distribution such as gaps and resonant clumps can reveal the presence and parameters of planets $[12,13]$.

At the same time, bright dust clouds constitute the main source of confusion and noise for future exoEarth imaging missions. The mere presence of dust emission (scattered light or thermal emission may dominate depending on the wavelength of observation) would add photon noise to the observations, even if the emission itself could be perfectly removed. Moreover, dust clumps due to resonant trapping by a planet can be misinterpreted as actual planets due to the limited angular resolution and signal-to-noise ratio of the observations. Thus, the expected amount of dust present around the target stars determines the design of any mission to detect exo-Earths, such as the required angular resolution and size of the primary mirror (e.g., $[14,15,16])$.

We have conducted the NASA funded HOSTS survey (Hunt for Observable Signatures of Terrestrial Systems) to search for habitable zone dust around a sample of nearby stars and to measure their dust levels. We observed in $N$ band, where the flux ratio between warm $(\sim 300 \mathrm{~K})$ dust and the star is most favorable while the atmosphere is still sufficiently transparent. The goal was to detect HZ dust levels only a few times more massive than the Solar system level, corresponding to an $N$ band excess emission of the order of $10^{-5}$. We thus used nulling interferometry at the Large Binocular Telescope Interferometer (LBTI, [17]) in order to spatially disentangle the dust emission from that of the star and to suppress the bright star light. 

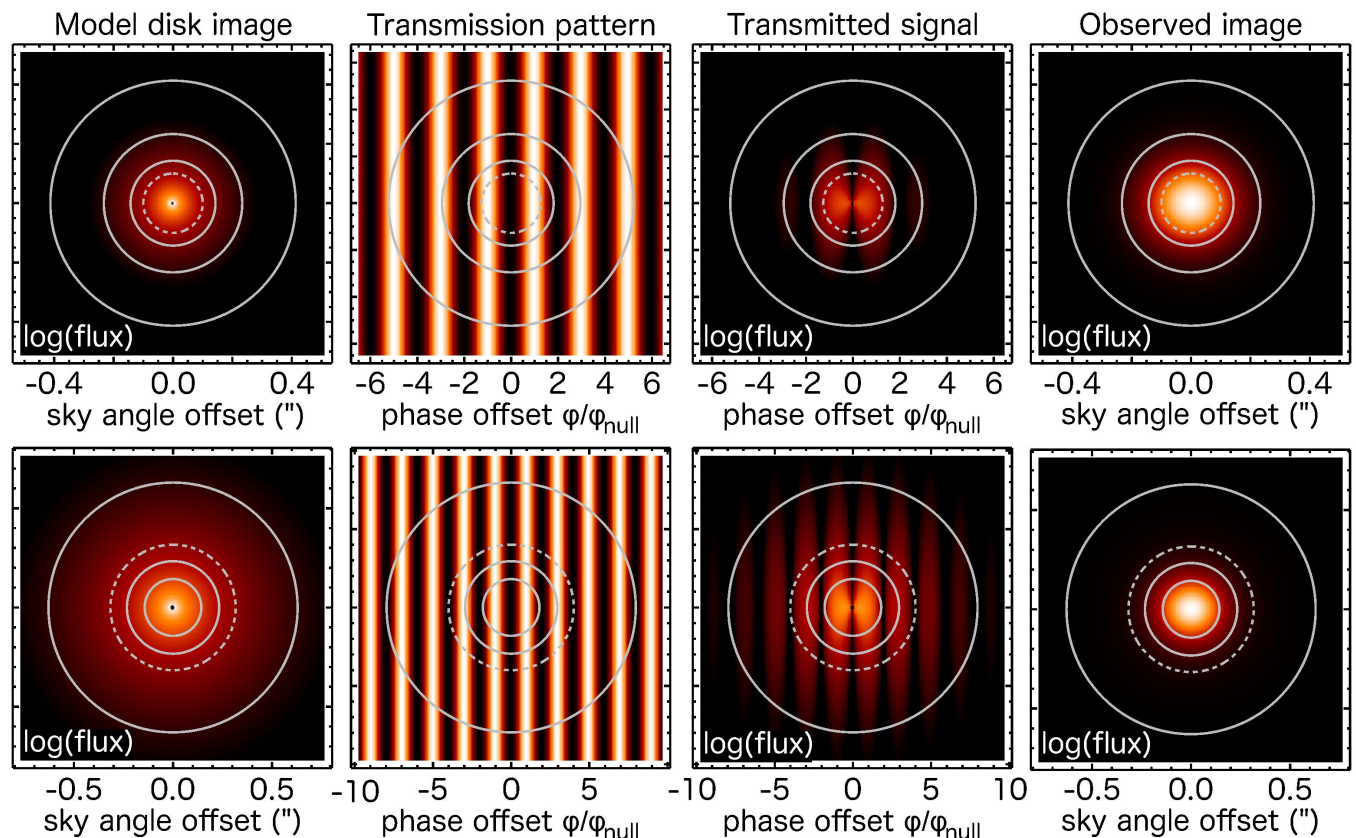

Figure 1. Illustration of the data obtained by nulling interferometry at the LBTI. Model observations are shown for two example stars (top row: $1 \mathrm{~L}_{\odot}$, bottom row: $10 \mathrm{~L} \odot$ ) at a distance of $10 \mathrm{pc}$. The panels show from left to right: A model image of a face-on disk, the LBTI transmission pattern, the transmission pattern applied to the model image, and the final simulated observation after convolving with the single aperture PSF. The disk models with and without the transmission pattern applied are shown in logarithmic scale, the other images are shown in linear scale. The dashed circle marks the size of the HZ (distance from the star receiving the same flux density as Earth from the Sun). The three solid circles mark three different photometric apertures used for the HOSTS survey. Simulations are shown, because this is typically not visible in real data due to various noise terms that can only be removed by a statistical analysis.

The survey observations have now been completed, but a fraction of the newest observations are still being analyzed. We discuss the latest results of our ongoing work and future prospects for the characterization of exozodiacal dust with the LBTI.

\section{OBSERVATIONS AND DATA ANALYSIS}

A detailed description of our observations and data reduction can be found in [3]. We present here only a brief summary.

Target stars have been selected for observations in real time from the full HOSTS target list presented by [18]. Our target stars are bright, nearby main sequence stars $\left(F_{\nu}>1\right.$ Jy in $N$ band) without known close $\left(<1.5^{\prime \prime}\right)$ binary companions. Spectral types range from A0 to K8. A list of observed stars with basic, relevant properties is given in Table 1. Calibrators were selected following [2] using the catalogs of [19] and [20], supplemented by stars from the JSDC catalog and the SearchCal tool (both [21]) where necessary.

The HOSTS survey was carried out with the LBTI on the Large Binocular Telescope (LBT) on Mt. Graham, Arizona. The wavefronts from the two $8.4 \mathrm{~m}$ apertures - separated by $14.4 \mathrm{~m}$ center to center - are stabilized using adaptive secondary mirrors. They are then brought to destructive interference in the LBTI and imaged on the Nulling Optimized Mid-Infrared Camera (NOMIC, [38]). Tip-tilt and optical path delay (OPD) tracking are performed using PhaseCam, a closed loop fringe tracker [39]. The adaptive optics system operates in the $R$ to $I$ band range, PhaseCam in the $K$ band, and nulling data are recorded in the $N$ band. The interferometric inner working angle is 70 mas. This is illustrated in Fig. 1. A nodding sequence is performed for sky subtraction and photometric observations of each star are obtained for relative flux calibration. Science observations (SCI) are paired with identical calibrator observations (CAL) to determine the nulling interferometric transfer function (TF) of the instrument. 
Table 1. Observed stars included in this work.

\begin{tabular}{|c|c|c|c|c|c|c|c|c|c|c|}
\hline $\begin{array}{c}\text { HD } \\
\text { number }\end{array}$ & Name & 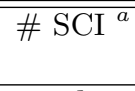 & Sp. Type & $\begin{array}{c}V \\
(\mathrm{mag}) \\
\end{array}$ & $\begin{array}{c}K \\
(\mathrm{mag}) \\
\end{array}$ & $\begin{array}{c}N^{\prime} \\
(\mathrm{Jy}) \\
\end{array}$ & $\begin{array}{c}d \\
(\mathrm{pc}) \\
\end{array}$ & $\begin{array}{l}\text { EEID } \\
(\mathrm{mas}) \\
\end{array}$ & $\begin{array}{c}\mathrm{fIR} / \mathrm{nIR} \\
\text { excess } \\
\end{array}$ & $\begin{array}{c}\text { Excess } \\
\text { references }\end{array}$ \\
\hline \multicolumn{11}{|c|}{ Sensitivity driven sample: } \\
\hline 33111 & $\beta$ Eri & 2 & A3 IV & 2.782 & 2.38 & 3.7 & 27.4 & 248 & $\mathrm{~N} / \mathrm{N}$ & {$[6,22,23]$} \\
\hline$* 38678$ & $\zeta$ Lep & 1 & A $2 \mathrm{IV}-\mathrm{V}$ & 3.536 & 3.31 & 2.1 & 21.6 & 176 & $\mathrm{Y} / \mathrm{N}$ & {$[6,22]$} \\
\hline *81937 & $23 \mathrm{UMa}$ & 5 & F0 IV & 3.644 & 2.73 & 2.6 & 23.8 & 168 & $\mathrm{~N} /-$ & {$[24]$} \\
\hline 95418 & $\beta \mathrm{UMa}$ & 4 & A1 IV & 2.341 & 2.38 & 4.2 & 24.5 & 316 & $\mathrm{Y} / \mathrm{N}$ & {$[5,25]$} \\
\hline 97603 & $\delta$ Leo & 4 & A5 IV & 2.549 & 2.26 & 3.9 & 17.9 & 278 & $\mathrm{~N} / \mathrm{N}$ & {$[5,22,23]$} \\
\hline 103287 & $\gamma \mathrm{UMa}$ & 4 & $\mathrm{~A} 0 \mathrm{~V}$ & 2.418 & 2.43 & 3.7 & 25.5 & 308 & $\mathrm{~N} /-$ & {$[22,23,25]$} \\
\hline 106591 & $\delta \mathrm{UMa}$ & 4 & $\mathrm{~A} 2 \mathrm{~V}$ & 3.295 & 3.10 & 2.0 & 24.7 & 199 & $\mathrm{~N} / \mathrm{N}$ & {$[5,22,23]$} \\
\hline 108767 & $\delta \mathrm{Crv}$ & 2 & A0 IV & 2.953 & 3.05 & 2.3 & 26.6 & 251 & $\mathrm{~N} / \mathrm{Y}$ & {$[6,22,23]$} \\
\hline 128167 & $\sigma$ Boo & 3 & $\mathrm{~F} 4 \mathrm{~V}$ & 4.467 & 3.47 & 1.4 & 15.8 & 117 & $\mathrm{Y} / \mathrm{N}$ & {$[5,22]$} \\
\hline 129502 & $\mu$ Vir & 3 & $\mathrm{~F} 2 \mathrm{~V}$ & 3.865 & 2.89 & 2.6 & 18.3 & 151 & $\mathrm{~N} / \mathrm{N}$ & {$[22,23]$} \\
\hline *172167 & $\alpha \operatorname{Lyr}$ & 4 & $\mathrm{~A} 0 \mathrm{~V}$ & 0.074 & 0.01 & 38.6 & 7.68 & 916 & $\mathrm{Y} / \mathrm{Y}$ & {$[4,25]$} \\
\hline 187642 & $\alpha \mathrm{Aql}$ & 2 & A7 V & 0.866 & 0.22 & 21.6 & 5.13 & 570 & $\mathrm{~N} / \mathrm{Y}$ & {$[5,22,23,26]$} \\
\hline 203280 & $\alpha$ Cep & 1 & $\mathrm{~A} 8 \mathrm{~V}$ & 2.456 & 1.85 & 7.0 & 15.0 & 294 & $\mathrm{~N} / \mathrm{Y}$ & {$[5,22,23,26]$} \\
\hline \multicolumn{11}{|c|}{ Sun like stars sample: } \\
\hline$* 9826$ & $\mu$ And & 2 & F9 V & 4.093 & 2.85 & 2.4 & 13.5 & 136 & $\mathrm{~N} / \mathrm{N}$ & {$[5,24,27]$} \\
\hline 10476 & 107 Psc & 3 & $\mathrm{~K} 1 \mathrm{~V}$ & 5.235 & 3.29 & 2.0 & 7.53 & 90 & $\mathrm{~N} / \mathrm{N}$ & {$[5,22,28,29]$} \\
\hline *10700 & $\tau$ Cet & 2 & G8 V & 3.489 & 1.68 & 5.4 & 3.65 & 182 & $\mathrm{Y} / \mathrm{Y}$ & {$[5,30]$} \\
\hline 16160 & GJ $105 \mathrm{~A}$ & 1 & $\mathrm{~K} 3 \mathrm{~V}$ & 5.815 & 3.45 & 1.5 & 7.18 & 73 & $\mathrm{~N} /-$ & {$[22,28,29]$} \\
\hline *22049 & $\epsilon$ Eri & 4 & $\mathrm{~K} 2 \mathrm{~V}$ & 3.721 & 1.66 & 7.4 & 3.22 & 172 & $\mathrm{Y} / \mathrm{N}$ & {$[5,31]$} \\
\hline *30652 & 1 Ori & 4 & $\mathrm{~F} 6 \mathrm{~V}$ & 3.183 & 2.08 & 4.8 & 8.07 & 205 & $\mathrm{~N} / \mathrm{N}$ & {$[5,22,28,29]$} \\
\hline 34411 & $\lambda$ Aur & 2 & G1 V & 4.684 & 3.27 & 1.8 & 12.6 & 105 & $\mathrm{~N} /-$ & {$[29,32]$} \\
\hline 48737 & $\xi \mathrm{Gem}$ & 3 & F5 IV-V & 3.336 & 2.13 & 4.3 & 18.0 & 196 & $-/ \mathrm{N}$ & {$[5]$} \\
\hline *78154 & $13 \mathrm{UMa}$ & 3 & F7 V & 4.809 & 3.53 & 1.2 & 20.4 & 99 & $\mathrm{~N} /-$ & {$[22]$} \\
\hline 88230 & GJ 380 & 2 & K8 V & 6.598 & 3.21 & 1.9 & 4.87 & 65 & $\mathrm{~N} /-$ & {$[27]$} \\
\hline 89449 & 40 Leo & 2 & F6 IV-V & 4.777 & 3.65 & 1.1 & 21.4 & 98 & $\mathrm{~N} /-$ & {$[22,24]$} \\
\hline$* 102870$ & $\beta \mathrm{Vir}$ & 4 & F9 V & 3.589 & 2.32 & 4.3 & 10.9 & 173 & $\mathrm{~N} / \mathrm{N}$ & {$[5,29]$} \\
\hline *120136 & $\tau$ Boo & 4 & F6 IV & 4.480 & 3.36 & 1.7 & 15.6 & 114 & $\mathrm{~N} / \mathrm{N}$ & {$[6,29,32]$} \\
\hline 126660 & $\theta$ Boo & 3 & F7 V & 4.040 & 2.81 & 3.1 & 14.5 & 147 & $\mathrm{~N} /-$ & {$[22,28,29]$} \\
\hline 141004 & $\lambda$ Ser & 2 & G0 IV-V & 4.413 & 2.98 & 2.4 & 12.1 & 121 & $\mathrm{~N} / \mathrm{N}$ & {$[5,22,28,33]$} \\
\hline 142373 & $\chi$ Her & 3 & G0 V & 4.605 & 3.12 & 2.0 & 15.9 & 111 & $\mathrm{~N} / \mathrm{N}$ & {$[5,22,24,28]$} \\
\hline 142860 & $\gamma$ Ser & 4 & F6 IV & 3.828 & 2.63 & 2.9 & 11.3 & 151 & $\mathrm{~N} / \mathrm{N}$ & {$[5,22,28,32]$} \\
\hline *173667 & 110 Her & 5 & $\mathrm{~F} 6 \mathrm{~V}$ & 4.202 & 3.03 & 2.2 & 19.2 & 131 & $\mathrm{Y} / \mathrm{Y}$ & {$[5,27]$} \\
\hline 185144 & $\sigma$ Dra & 2 & G9 V & 4.664 & 2.83 & 2.7 & 5.76 & 113 & $\mathrm{~N} / \mathrm{N}$ & {$[5,29,32]$} \\
\hline 215648 & $\xi \operatorname{Peg} \mathrm{A}$ & 3 & $\mathrm{~F} 6 \mathrm{~V}$ & 4.203 & 2.90 & 2.2 & 16.3 & 132 & $\mathrm{~N} / \mathrm{N}$ & {$[22,24,28]$} \\
\hline *222368 & $\iota \mathrm{Psc}$ & 2 & F7 V & 4.126 & 2.83 & 2.4 & 13.7 & 137 & $\mathrm{~N} /-$ & {$[22,24,28]$} \\
\hline \multicolumn{11}{|c|}{ Commissioning targets: } \\
\hline 102647 & $\beta$ Leo & 2 & A3 V & 2.121 & 1.92 & 6.9 & 11.0 & 336 & $\mathrm{Y} / \mathrm{Y}$ & {$[5,25]$} \\
\hline 109085 & $\eta \mathrm{Crv}$ & 3 & $\mathrm{~F} 2 \mathrm{~V}$ & 4.302 & 3.54 & 1.8 & 18.3 & 125 & $\mathrm{Y} / \mathrm{N}$ & {$[5,34]$} \\
\hline
\end{tabular}

The full list of stars observed by the HOSTS survey and all null measurements and derived zodi levels will be published in a dedicated paper (Ertel et al., in prep.). Stars with new observations since [3] are marked with an asterisk. EEID is the Earth Equivalent Insolation Distance, the distance at which a body receives the same flux density from its host star as Earth from the Sun. Magnitudes are given in the Vega system.

$a$ Number of calibrated science pointings obtained. References are: Spectral type: SIMBAD; $V$ magnitude: [35]; $K$ magnitude: [36] and the Lausanne photometric data base (http://obswww.unige.ch/gcpd/gcpd.html); $N$ band flux and EEID: [18]; Distance: [37] 

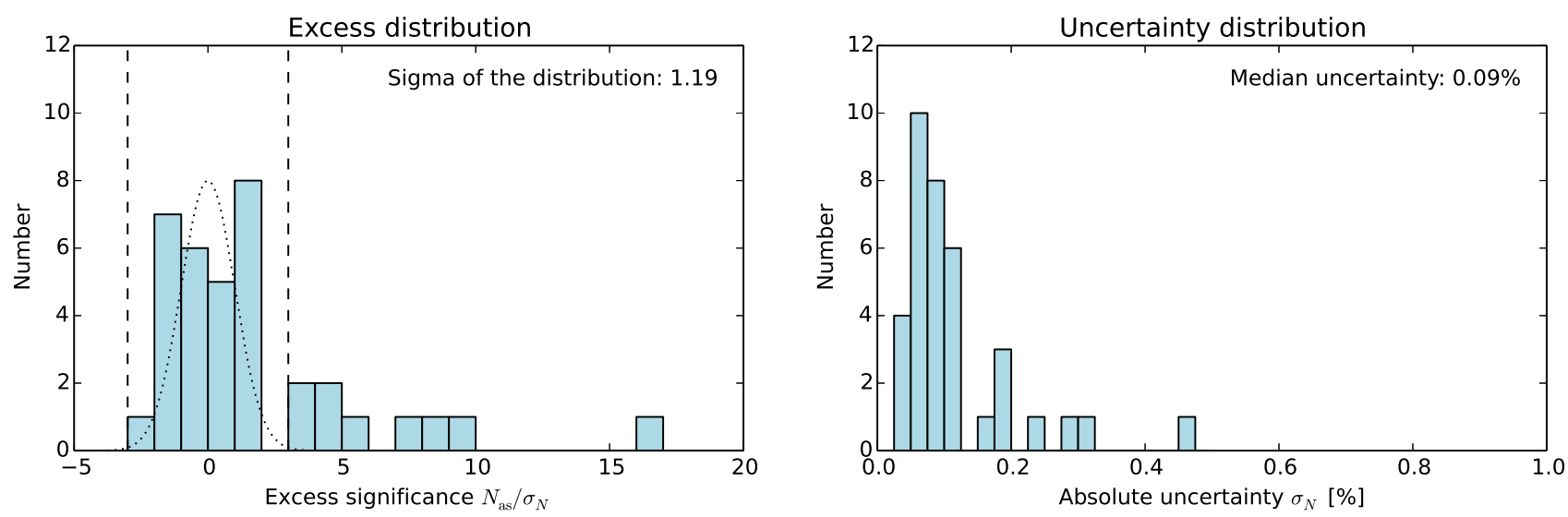

Figure 2. Distribution of excess significance $N_{\mathrm{as}} / \sigma_{N}$ (left) and uncertainties $\sigma_{N}$ (right). The two vertical, dashed lines in the excess significance distribution plots mark the $\pm 3 \sigma$ boundaries based on our uncertainty estimates. The standard deviation of the distribution is computed from non-detections only ( $-3<N_{\text {as }} / \sigma_{N}<3$ ). The dotted line represents a Gaussian with a standard deviation of one (Normal distribution) scaled to the peak of the histogram and is used only to guide the eye.

After a basic reduction of each frame (nod subtraction, bad pixel correction), photometry is performed on each single frame. The raw null depth and its uncertainty are determined using the null self calibration (NSC, $[40,41,42,43])$. Null measurements are converted to dust levels using a power-law radial dust distribution model analogous to the Solar system's zodiacal dust $[44,45]$. The vertical optical depth of the dust at a separation of 1 AU from the Sun of $7.12 \times 10^{-8}$ defines our unit of 1 zodi.

The beam combination in the $N$ band is achieved in the pupil plane before the light is re-imaged in the focal plane. A consequence is that the light from a point source has the same phase over the entire detector, but the phase is different at different sky angles. This results in a spatial filtering in form of a transmission pattern (stripes of high and low transmission vertical to the baseline orientation) being applied to the flux distribution on sky (Fig. 1).

\section{RESULTS}

\subsection{Excess significance and detection threshold}

Fig. 2 shows the distributions of the excess significance $N_{\mathrm{as}} / \sigma_{N}$ and of the uncertainties. The distributions are in general well behaved with $N_{\text {as }} / \sigma_{N}$ following a Normal distribution for $-3<N_{\text {as }} / \sigma_{N}<3$ with the addition of several detections at $N_{\text {as }} / \sigma_{N}>3$. We apply a $3 \sigma$ threshold to identify significant excesses. We find ten significant excess detections.

\subsection{Updates on specific targets}

Seven of our ten detections have already been discussed in detail by [3]. Among those, no new data are included in this work for $\beta$ Leo, $\eta \mathrm{Crv}, \beta \mathrm{UMa}, \delta \mathrm{UMa}$, and $\theta$ Boo. New data have been taken and the results are included here for $\epsilon$ Eri and 110 Her. Both detections were originally only achieved with a very large aperture size and at relatively low significance. They thus required follow-up observations. For $\epsilon$ Eri we confirm our detection with new, high accuracy data and measure a zodi level of $368 \pm 68$ zodis. A detection is also achieved in a smaller aperture (reduced background and read noise). The detection around 110 Her is also confirmed with a new measurement of $343 \pm 57$ zodis. This detection is, however, still only achieved in a large aperture, with strong limits for smaller apertures, suggesting that the dust may be concentrated at a relatively large separation from the star. As discussed already by [3] the low significance detection of far-infrared excess around this star is controversial, so that it is not clear whether to include it in the group of stars with or without previously detected cold dust. 


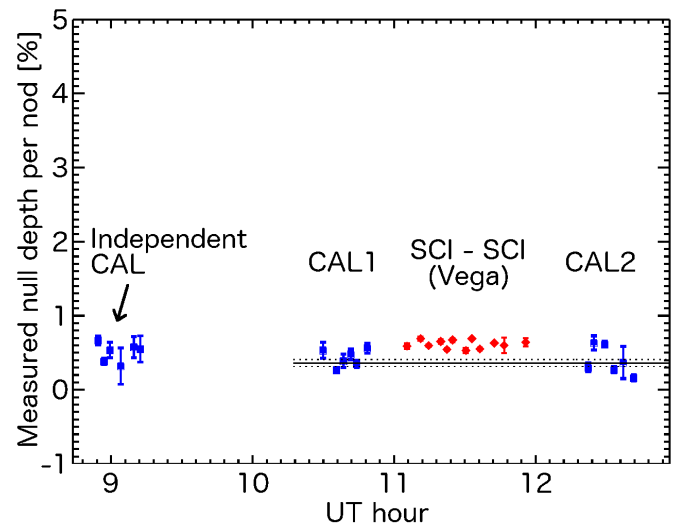

Figure 3. A HOSTS observing sequence (CAL-SCI-SCI-CAL) on Vega showing the faint (4.5 $\sigma$ excess). The blue points are calibrator observations, the red points are science observations. Measurements from each single nod are displayed. The small vertical offset between science and calibrator measurements is the null excess. The first calibrator shown is an unrelated observation of a star at a different sky location. The data illustrate the high instrument stability both over time and over pointing direction.

In the new data obtained since summer 2017 (not included in [3]), we find three new detections: The Sun-like star $13 \mathrm{UMa}$ shows a very strong excess of $550 \pm 66$ zodis. After $\delta \mathrm{UMa}$ and $\theta$ Boo, this is the third star in our survey without previously known cold excess, but with an LBTI detection (potentially the fourth one when including 110 Her, see above). This further supports our conclusion in [3] that focussing on stars without known cold dust for exo-Earth imaging does not guarantee a low HZ dust level. We further confirm the detection of HZ dust around $\zeta$ Lep by [2] and measure a zodi level of $739 \pm 46$ zodis.

Most interesting is the fact that new data obtained show a detection of $38 \pm 8$ zodis around Vega. Our new observations achieved a better sensitivity and the detection is consistent with the previous upper limit presented in [3]. The excess is the strongest in a large aperture and not detected in a very small one, which means we are not seeing the hot dust in this system previously reported by [4], but rather dust further out, near the HZ. An observing sequence on Vega is shown in Fig. 3.

Finally, our upper limit on $\tau$ Cet of 74 zodis $(3 \sigma)$ is noteworthy. This star hosts a known debris disk (e.g., [46]), hot dust [5], and four claimed super-Earth mass planets ( $\tau$ Cet e, f, g, h, [47]) with $\tau$ Cet f being in the $\mathrm{HZ}$, but also at sufficient angular separation that it might be detectable with a coronagraph on WFIRST. Our upper limit shows that despite the high dust content in the system at large and very small separations, the HZ is relatively dust poor.

\subsection{Statistical results}

In this section, we provide an update of the statistical analysis from [3] considering our additional data.

We divide our sample into early type stars and Sun-like stars, and into stars with previously known cold dust ('dusty stars') and without ('clean stars'). As in [3] we exclude $\eta$ Crv and $\beta$ Leo from the statistical analysis as biased targets. Detection rates for these subsamples are listed in Table 2 and shown in Fig. 4. The results of Fisher's exact test to check if differences in the detection rates are significant are shown in Table 3. We can rule out with high confidence (probability $0.3 \%$ ) that the occurrence rate of $\mathrm{HZ}$ dust is the same among clean and dusty stars. Note that this result is affected by small number statistics of detections often at the boarder of significance, both in our mid-infrared nulling data and in the far-infrared. For example, moving 110 Her into the category of stars without a significant cold dust detection, this result moves to a considerably lower significance probability changes to 0.017 . The probability for having the same occurrence rate for stars with and without known cold dust among Sun-like stars only is nominally 0.08, and becomes 0.32 when considering 110 Her as a star without cold dust. We can thus not claim a significant correlation between cold and warm dust for Sun-like stars and the correlation for all stars needs to be treated with care. The occurrence rates for Sun-like and 


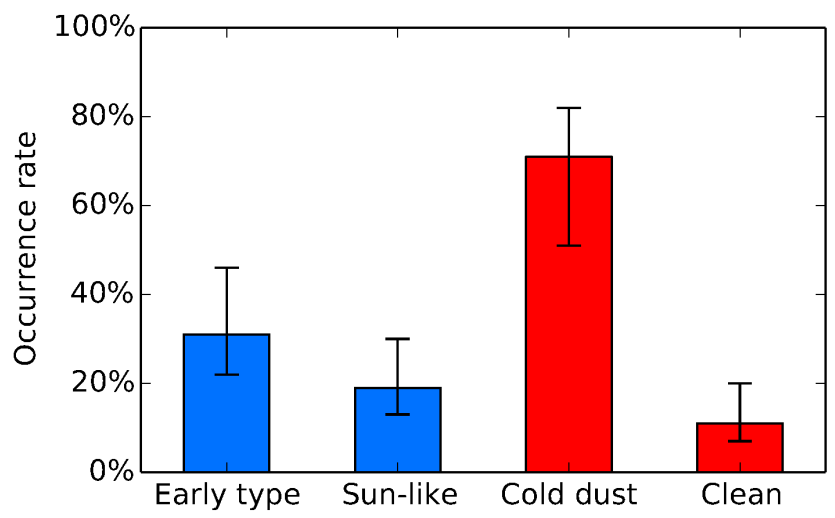

Figure 4. Occurrence rates of exozodiacal dust inferred from the observations considered in this work for early-type stars, Sun-like stars, stars with previously known cold dust, and stars without detected cold dust.

Table 2. Subsamples, excess detections, and occurrence rates

\begin{tabular}{cccc}
\hline \hline & Cold dust & Clean & All \\
\hline Early & 3 of 4 & 1 of 9 & 4 of 13 \\
type & $75_{-27}^{+10} \%$ & $11_{-4}^{+18} \%$ & $31_{-9}^{+15} \%$ \\
Sun- & 2 of 3 & 2 of 18 & 4 of 21 \\
like & $67_{-28}^{+15 \%}$ & $11_{-4}^{+12} \%$ & $19_{-6}^{+11} \%$ \\
All & 5 of 7 & 3 of 27 & 8 of 34 \\
& $71_{-20}^{+11} \%$ & $11_{-4}^{+9} \%$ & $23_{-6}^{+9} \%$ \\
\hline
\end{tabular}

Table 3. Probability that two samples are drawn from the same distribution

\begin{tabular}{ccc}
\hline \hline Samples 1 & Sample 2 & Probability \\
\hline All early type & All Sun-like & 0.24 \\
All dusty & All clean & 0.003 \\
Clean early type & Dusty early type & 0.05 \\
Clean Sun-like & Dusty Sun-like & 0.08 \\
Clean early type & Clean Sun-like & 0.47 \\
Dusty early type & Dusty Sun-like & 0.57 \\
\hline
\end{tabular}

early-type stars are the same within the binomial uncertainties. This is surprising given the typically $\sim 4$ times lower sensitivity to HZ dust surface density around Sun-like compared to early-type stars [18, 45]).

Next, we repeat the statistical analysis presented by [3] to determine the typical HZ dust levels around our target stars, including the newest data available. We fit a lognormal exozodi luminosity function with parameters $\mu$ and $\varsigma$ to our measurements using a maximum likelihood estimate and performing a Bayesian analysis. We apply a $1 / m$ prior, equivalent to assuming a flat prior in $\mu$, marginalize the likelihood distribution over $\varsigma$, and compute the posterior cumulative probability distribution function (CPDF) of $m=\exp (\mu)$. For Sun-like stars without previously detected cold dust, we currently find a 95\% confidence upper limit on the median zodi level of 16 zodis. These stars are the highest priority targets for future exo-Earth imaging missions.

\section{FUTURE PROSPECTS}

\subsection{Extending HOSTS and target vetting for future exo-Earth imaging}

This summer 2018, the LBTI will undergo an adaptive optics upgrade designed to improve its wavefront stability and performance at low target elevation. In addition, we are improving our application of the NSC to improve background subtraction (currently a dominant source of uncertainty) with minimal change to the data acquisition 


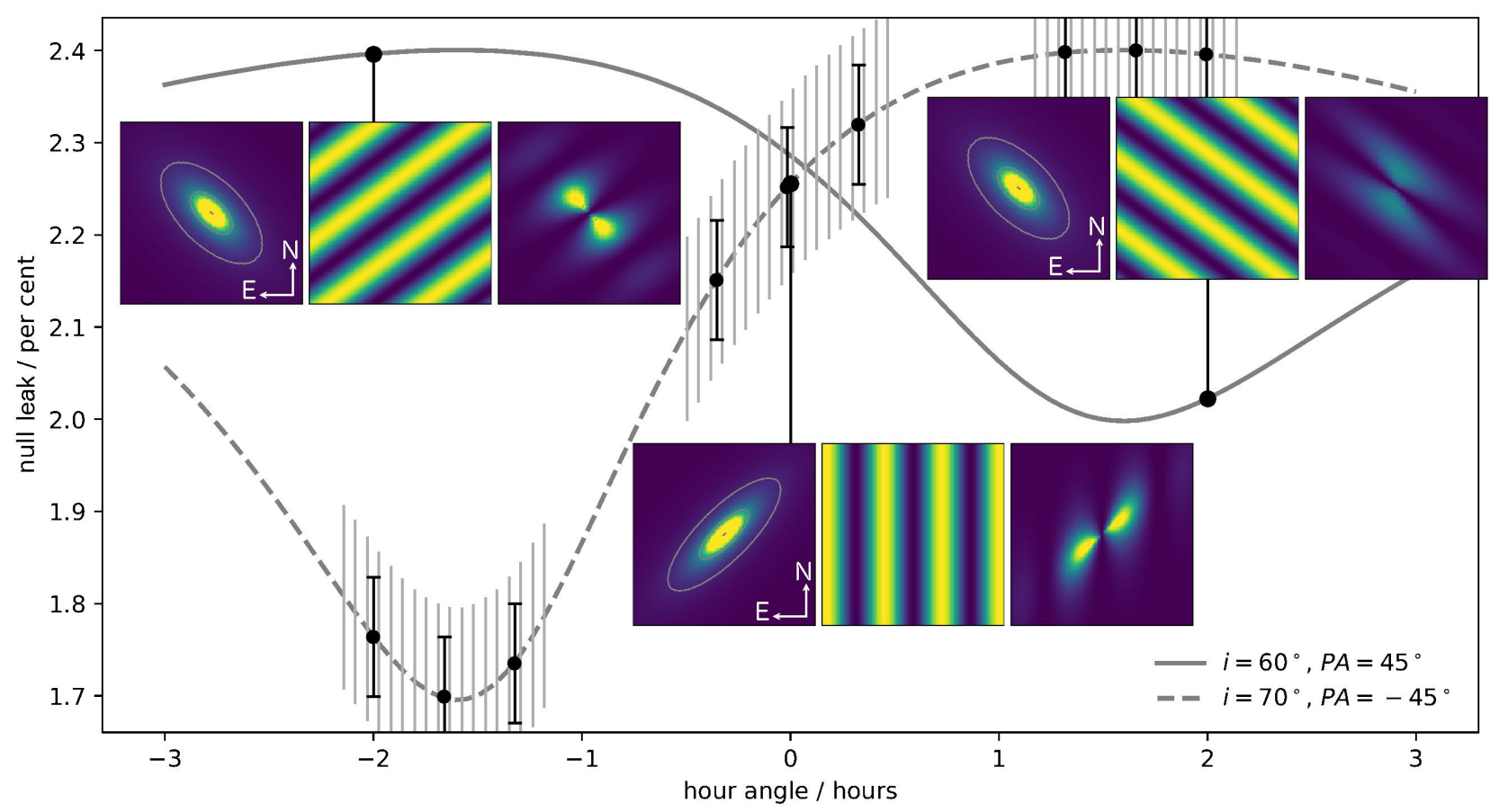

Figure 5. Simulation of observed null leak vs. hour angle of observation for two disks with different position angles and inclinations. The disk model, the transmission pattern, and the transmitted signal are shown for three representative cases. Over plotted on the $i=70 \mathrm{deg}$ curve is a planned observing sequence and uncertainties for the proposed observations. The high cadence, gray bars show the single nods and relative errors (relevant to detect variations over HA), while the black points and uncertainties show the measurement when combining 6 nods including absolute calibrations (relevant when combining observations from different nights). The large gaps are due to calibrator observations. Our detection around $\beta \mathrm{UMa}$ is used as a template.

strategy. With the improved instrument performance and sensitivity, new survey observations will provide even stronger statistical results.

Furthermore, our detections around $\theta$ Boo and $13 \mathrm{UMa}$ (both Sun-like stars without known cold dust) show that selecting such stars for exo-Earth imaging does not guarantee low HZ dust levels. One option to improve over this selection strategy is to perform target vetting with the LBTI and a suitable future instrument in the South $[48,49]$. To illustrate the improvement possible with such a strategy, we produce from our sample of Sun-like stars without cold dust an LBTI vetted sample by excluding the HOSTS detections. For such a sample we find an upper limit on the median zodi level of 7.5 zodis, an improvement by a factor of two. This translates in a $20 \%$ increase in yield for an exo-Earth imaging mission of a given size [14].

\subsection{Characterization of detected systems using nulling interferometry}

The performance of LBTI for the HOSTS survey has reached a level that makes nulling infrared interferometry a powerful tool to study warm dust systems around nearby main sequence stars. With a detection rate of the HOSTS survey of $\sim 25 \%$ and a sensitivity of only a few zodis for our most favorable stars, we can now study systems with typical HZ dust levels for the first time. Our high signal-to-noise detections allow for detailed follow-up observations.

The alt-azimuth mount of the LBTI allows us to measure the excess at different position angles of the single interferometric baseline projected on sky when observing at different hour angles. A different disk size along different position angles (an inclined disk) or disk structure cause variation of the null excess with baseline orientation. This allows us to measure the inclination and position angle of the disk, and to search for structures due to planet-disk interaction. The latter will rotate with the orbital period of the responsible planet, which is 
of the order of a few years for planets close to the HZ. Thus, monitoring over a few years can be used to to better distinguish between different scenarios for the hour angle variation and to constrain the orbit of the responsible planet. In addition, observations at different wavelengths across the $N$ band provide different spatial filtering by the transmission pattern, thus further constraining the geometry of the emission. Multi-wavelength data also allow us to constrain the spectral shape of the excess emission.

These information allow us to address the most pressing questions on exozodiacal dust after the HOSTS survey. Determining the geometry and radial dust distribution is critical for an accurate determination of the zodi level from our nulling data. Information on the radial dust distribution for a range of systems with different zodi levels and stellar spectral types will also allow us to make better estimates of the probable levels of exozodi in systems where it has not been detected. This will allow us to derive more reliable upper limits and thus more reliable statistics from the HOSTS survey. The radial slope of the dust distribution can inform us about the dust origin: Poynting-Robertson drag from an outer disk would result in a flat or inwardly decreasing surface density distribution [10], while a cometary origin would produce an inwardly increasing one [50, 51]. The detection of potential disk structures and radial discontinuities of the surface density distribution allows us to put constraints on the responsible planets (orbit, mass, [12, 13], Bonsor et al., in prep.). This provides present-day insight into the architecture and dynamics of nearby planetary systems in and near their HZs. Information on the spectral slope of the dust emission allows us to constrain the dust grain size [52] and thus its origin (Poynting-Robertson drag, local collisional production, or cometary origin). Moreover, a better understanding of the dust grain size allows us to better predict its scattered light brightness [53]. This is critical to better understand the implications of our HOSTS results (obtained in the mid-infrared) for exo-Earth imaging (to be achieved in the visible).

We demonstrate the feasibility of the proposed observations using simulations of the expected signal for several scenarios, using our detection around $\beta$ UMa as a template. Fig. 5 shows the null excess vs. hour angle of observations for two disks at different inclinations and position angles. It can be seen that the null excess varies by $\sim 20 \%$ and $\sim 35 \%$ for the two examples due to the disk inclination. Fig. 6 shows the structure produced by a $20 \mathrm{M}_{\oplus}$ planet at $1 \mathrm{AU}$ from a Sun-like star in dust spiraling inward from an asteroid belt at $2.25 \mathrm{AU}$ due to Poynting-Robertson drag [13] and the expected null variation vs. position angle of LBTI's transmission pattern. Again, a variation of $\sim 20 \%$ can be expected. LBTI's current accuracy to null excess is $\sim 6 \times 10^{-4}(1 \sigma)$ for a calibrated observing sequence. Thus, the expected signals can be detected with two observations in the extrema of the null variation if the mean excess is $\sim 1 \%$. They should thus be well detectable in our systems with the strongest excesses ( $\beta$ Leo, $\beta$ UMa, $\eta \mathrm{Crv}, \zeta$ Lep, $13 \mathrm{UMa}$ ). In addition, strong structures on top of the measured excesses may be visible for our other detections including $\epsilon$ Eri and Vega. Because the hour angles of the extrema of the null excess are unknown a priori, observations need to be carried out at a range of hour angles. This will naturally produce more data than observations in the extrema only and thus yield a higher sensitivity to the observed features. A realistic observing cadence and hour angle coverage of observations optimized to detect variations of the null excess with hour angle is shown in Fig. 5.

Simulations at two wavelengths (e.g., $7.9 \mu \mathrm{m}$ and $11.9 \mu \mathrm{m}$, filter width $\sim 10 \%$ ) of a face-on disk with two different surface density slopes (flat and outward decreasing with $1 / r$ ) are shown in Fig. 7 . From these data one can measure the null excess with different photometric aperture sizes to reconstruct its angular size which is at least marginally resolved by the single aperture PSF. Moreover, one can measure the null excess ratio between two wavelengths, which is $N_{11.9} / N_{7.9}=1.4$ and $N_{11.9} / N_{7.9}=0.9$ for the flat and $1 / r$ surface density cases, respectively. This difference is also detectable for null excesses around $1 \%$ with single observations in each wavelength, and for lower excesses if more data are obtained.

Detailed model fitting, combining the wealth of data provided by the observations described above can also break degeneracies in single data sets $[45,52,53,54]$. Such degeneracies are for example present between disk inclination and structure as a source of hour angle variation of the measured excess or between dust grain size and radial distribution in the wavelength dependence of the measured null excesses. However, the first can be broken when measuring the variation at two wavelengths (the inclination effect is related to the inner working angle, the effect of a clump typically not). The latter can be broken by measuring the radial distribution also with different photometric aperture sizes. 

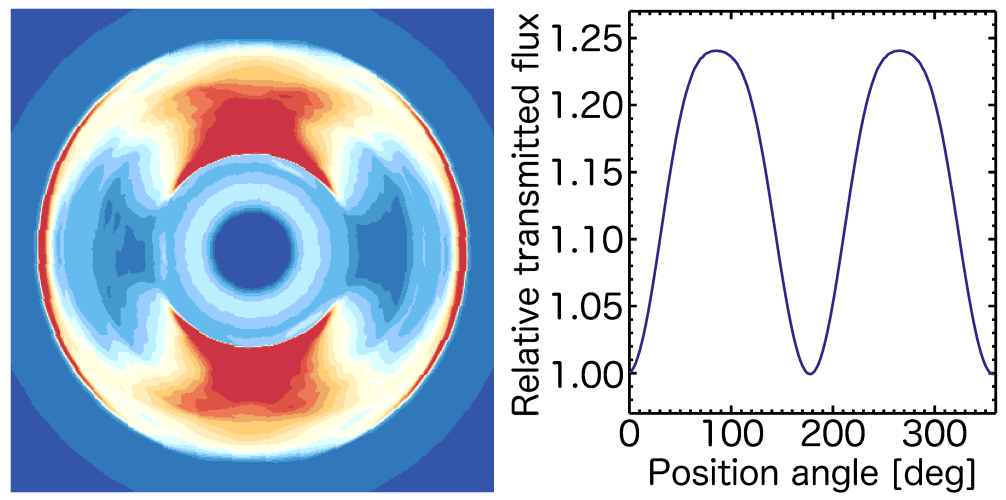

Figure 6. Simulation of observed null leak vs. hour angle (right) for an observation of a disk with resonant structures due to planet-disk-interaction (left, [13]). The dust surface density is shown in the left image, where red corresponds to high and blue to low surface density. Simulations have been performed for a $20 \mathrm{M}_{\oplus}$ planet at a separation of 1 au from a Sun-like star (distance $10 \mathrm{pc}$ ).

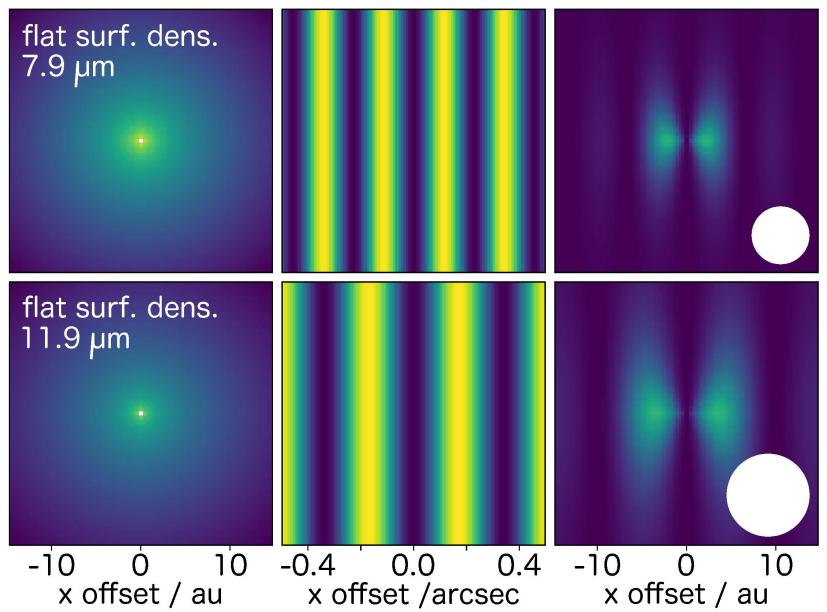

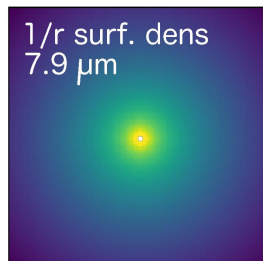
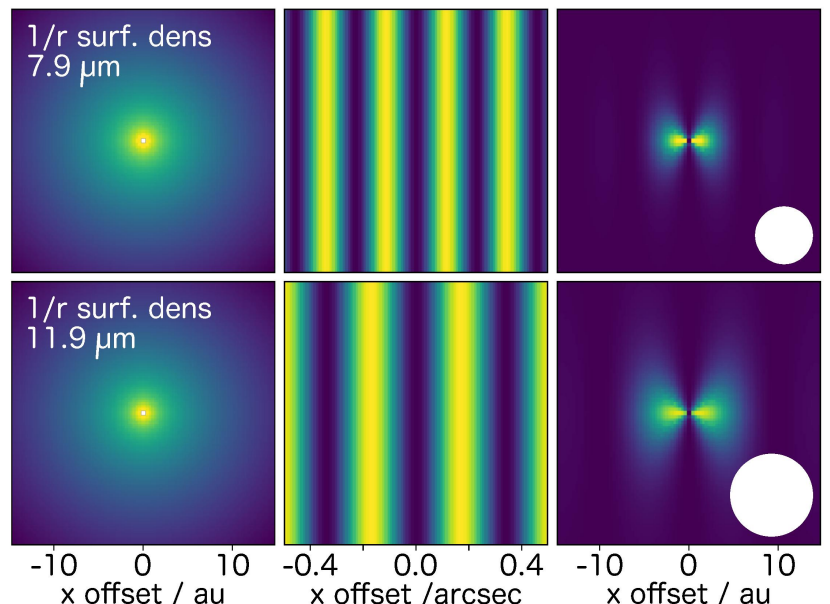

Figure 7. Transmission of disk flux at $7.9 \mu \mathrm{m}$ ( top row) and $11.9 \mu \mathrm{m}$ (bottom row) for two disk models with different radial surface density (left three columns: flat, right three: $1 / r$ ). The disk model image (in logarithmic scale), transmission pattern, and transmitted flux are shown. All disk model images are shown in the same color scale. Transmitted flux is shown in the same color scale for the same wavelength but the peak brightness is twice as high at $7.9 \mu \mathrm{m}$ than at $11.9 \mu \mathrm{m}$. The flux ratio of the null excess at $11.9 \mu \mathrm{m}$ and $7.9 \mu \mathrm{m}$ is $N_{11.9} / N_{7.9}=1.4$ for the flat surface density and $N_{11.9} / N_{7.9}=0.9$ for the $1 / r$ case. The white circles illustrate the FWHM of the single telescope PSF. It can be seen that the transmitted flux is resolved. Our detection of $\beta$ UMa is used as a template.

\subsection{Characterization of detected systems using Fizeau spectro-interferometry}

Another possibility the LBTI offers for the characterization of exozodiacal dust is spectro-interferometry in the $K$ to $N$ band in constructive Fizeau mode. The LBTI is currently the only interferometer operating in this wavelength range and using a fringe tracker. Thus, it should in principle be possible to obtain spectrally resolved data with an accuracy sufficient to detect excesses at the $\sim 1 \%$ level. Such data could for example be used to study the connection between HZ dust and hot dust closer in by tracing the warm dust emission toward shorter wavelengths of the hot emission toward longer wavelengths. It should also be possible to detect the $3 \mu \mathrm{m}$ and $10 \mu \mathrm{m}$ silicate features of the dust to further study its composition. A description of LBTI's Fizeau mode and our efforts in implementing routine, accurate, and efficient observations is presented by [55].

\section{CONCLUSIONS}

We have conducted the most sensitive survey for HZ dust around nearby stars. We put strong constraints on the typical HZ dust levels and show that these are low enough for stars without known cold dust to allow exo-Earth 
imaging. However, our detections around these stars demonstrate that such a target selection strategy does not guarantee low HZ dust levels for all stars. This limits our constraint on the median dust level to 16 zodis, while for a sample of LBTI vetted stars the median level would be by a factor of two better. This transforms into a $20 \%$ increase of the yield of an exo-Earth imaging mission of a given size. We have also outlined the science case for a future characterization of the HOSTS detected exozodis with the LBTI and demonstrated the feasibility of the proposed observations through modeling of the expected signals. These observations represent a critical next step in the understanding of the origin and properties of exozodiacal dust and provide present-day insights into the architecture and dynamics of nearby planetary systems near their HZs.

\section{ACKNOWLEDGMENTS}

The Large Binocular Telescope Interferometer is funded by the National Aeronautics and Space Administration as part of its Exoplanet Exploration Program. The LBT is an international collaboration among institutions in the United States, Italy, and Germany. LBT Corporation partners are: The University of Arizona on behalf of the Arizona university system; Instituto Nazionale di Astrofisica, Italy; LBT Beteiligungsgesellschaft, Germany, representing the Max-Planck Society, the Astrophysical Institute Potsdam, and Heidelberg University; The Ohio State University, and The Research Corporation, on behalf of The University of Notre Dame, University of Minnesota and University of Virginia. This research has made extensive use of the SIMBAD database [56] and the VizieR catalogue access tool [57], both operated at CDS, Strasbourg, France, of Python, including the NumPy, SciPy, Matplotlib [58], and Astorpy [59] libraries, and of NASA's Astrophysics Data System Bibliographic Services. GMK is supported by the Royal Society as a Royal Society University Research Fellow. AS is partially supported by funding from the Center for Exoplanets and Habitable Worlds. The Center for Exoplanets and Habitable Worlds is supported by the Pennsylvania State University, the Eberly College of Science, and the Pennsylvania Space Grant Consortium. JMS is supported by NASA through Hubble Fellowship grant HSTHF2-51398.001-A awarded by the Space Telescope Science Institute, which is operated by the Association of Universities for Research in Astronomy, Inc., for NASA, under contract NAS5-26555.

\section{References}

[1] Millan-Gabet, R., Serabyn, E., Mennesson, B., Stark, C. C., Ragland, S., Hrynevych, M., Woillez, J., Stapelfeldt, K., Bryden, G., Colavita, M. M., and Booth, A. J., "Exozodiacal Dust Levels for Nearby Main-sequence Stars: A Survey with the Keck Interferometer Nuller," ApJ 734, 67 (June 2011).

[2] Mennesson, B., Millan-Gabet, R., Serabyn, E., Colavita, M. M., Absil, O., Bryden, G., Wyatt, M., Danchi, W., Defrère, D., Doré, O., Hinz, P., Kuchner, M., Ragland, S., Scott, N., Stapelfeldt, K., Traub, W., and Woillez, J., "Constraining the Exozodiacal Luminosity Function of Main-sequence Stars: Complete Results from the Keck Nuller Mid-infrared Surveys," ApJ 797, 119 (Dec. 2014).

[3] Ertel, S., Defrère, D., Hinz, P., Mennesson, B., Kennedy, G. M., Danchi, W. C., Gelino, C., Hill, J. M., Hoffmann, W. F., Rieke, G., Shannon, A., Spalding, E., Stone, J. M., Vaz, A., Weinberger, A. J., Willems, P., Absil, O., Arbo, P., Bailey, V. P., Beichman, C., Bryden, G., Downey, E. C., Durney, O., Esposito, S., Gaspar, A., Grenz, P., Haniff, C. A., Leisenring, J. M., Marion, L., McMahon, T. J., Millan-Gabet, R., Montoya, M., Morzinski, K. M., Pinna, E., Power, J., Puglisi, A., Roberge, A., Serabyn, E., Skemer, A. J., Stapelfeldt, K., Su, K. Y. L., Vaitheeswaran, V., and Wyatt, M. C., "The HOSTS Survey - Exozodiacal Dust Measurements for 30 Stars," AJ 155, 194 (May 2018).

[4] Absil, O., di Folco, E., Mérand, A., Augereau, J.-C., Coudé du Foresto, V., Aufdenberg, J. P., Kervella, P., Ridgway, S. T., Berger, D. H., ten Brummelaar, T. A., Sturmann, J., Sturmann, L., Turner, N. H., and McAlister, H. A., "Circumstellar material in the ¡ASTROBJ ¿Vegai/ASTROBJ $i$ inner system revealed by CHARA/FLUOR," AESA 452, 237-244 (June 2006).

[5] Absil, O., Defrère, D., Coudé du Foresto, V., Di Folco, E., Mérand, A., Augereau, J.-C., Ertel, S., Hanot, C., Kervella, P., Mollier, B., Scott, N., Che, X., Monnier, J. D., Thureau, N., Tuthill, P. G., ten Brummelaar, T. A., McAlister, H. A., Sturmann, J., Sturmann, L., and Turner, N., "A near-infrared interferometric survey of debris-disc stars. III. First statistics based on 42 stars observed with CHARA/FLUOR," A 6 A 555, A104 (July 2013). 
[6] Ertel, S., Absil, O., Defrère, D., Le Bouquin, J.-B., Augereau, J.-C., Marion, L., Blind, N., Bonsor, A., Bryden, G., Lebreton, J., and Milli, J., "A near-infrared interferometric survey of debris-disk stars. IV. An unbiased sample of 92 southern stars observed in $\mathrm{H}$ band with VLTI/PIONIER," A $6 A$ 570, A128 (Oct. 2014).

[7] Ertel, S., Defrère, D., Absil, O., Le Bouquin, J.-B., Augereau, J.-C., Berger, J.-P., Blind, N., Bonsor, A., Lagrange, A.-M., Lebreton, J., Marion, L., Milli, J., and Olofsson, J., "A near-infrared interferometric survey of debris-disc stars. V. PIONIER search for variability," A\&A 595, A44 (Oct. 2016).

[8] Faramaz, V., Ertel, S., Booth, M., Cuadra, J., and Simmonds, C., "Inner mean-motion resonances with eccentric planets: a possible origin for exozodiacal dust clouds," MNRAS 465, 2352-2365 (Feb. 2017).

[9] Backman, D. E. and Paresce, F., "Main-sequence stars with circumstellar solid material - The VEGA phenomenon," in [Protostars and Planets III], E. H. Levy \& J. I. Lunine, ed., 1253-1304 (1993).

[10] Kennedy, G. M. and Piette, A., "Warm exo-Zodi from cool exo-Kuiper belts: the significance of P-R drag and the inference of intervening planets," MNRAS 449, 2304-2311 (May 2015).

[11] Reidemeister, M., Krivov, A. V., Stark, C. C., Augereau, J., Löhne, T., and Müller, S., "The cold origin of the warm dust around $\epsilon$ Eridani," A $\& A$ 527, A57+ (Mar. 2011).

[12] Ertel, S., Wolf, S., and Rodmann, J., "Observing planet-disk interaction in debris disks," A\&A 544, A61 (Aug. 2012).

[13] Shannon, A., Mustill, A. J., and Wyatt, M., "Capture and evolution of dust in planetary mean-motion resonances: a fast, semi-analytic method for generating resonantly trapped disc images," MNRAS 448, 684-702 (Mar. 2015).

[14] Stark, C. C., Roberge, A., Mandell, A., Clampin, M., Domagal-Goldman, S. D., McElwain, M. W., and Stapelfeldt, K. R., "Lower Limits on Aperture Size for an ExoEarth Detecting Coronagraphic Mission," ApJ 808, 149 (Aug. 2015).

[15] Stark, C. C., Shaklan, S., Lisman, D., Cady, E., Savransky, D., Roberge, A., and Mandell, A. M., "Maximized exoEarth candidate yields for starshades," Journal of Astronomical Telescopes, Instruments, and Systems 2, 041204 (Oct. 2016).

[16] Defrère, D., Absil, O., den Hartog, R., Hanot, C., and Stark, C., "Nulling interferometry: impact of exozodiacal clouds on the performance of future life-finding space missions," A\&SA 509, A9 (Jan. 2010).

[17] Hinz, P. M., Defrère, D., Skemer, A., Bailey, V., Stone, J., Spalding, E., Vaz, A., Pinna, E., Puglisi, A., Esposito, S., Montoya, M., Downey, E., Leisenring, J., Durney, O., Hoffmann, W., Hill, J., Millan-Gabet, R., Mennesson, B., Danchi, W., Morzinski, K., Grenz, P., Skrutskie, M., and Ertel, S., "Overview of LBTI: a multipurpose facility for high spatial resolution observations," in [Optical and Infrared Interferometry and Imaging V], Proc. SPIE 9907, 990704 (Aug. 2016).

[18] Weinberger, A. J., Bryden, G., Kennedy, G. M., Roberge, A., Defrère, D., Hinz, P. M., Millan-Gabet, R., Rieke, G., Bailey, V. P., Danchi, W. C., Haniff, C., Mennesson, B., Serabyn, E., Skemer, A. J., Stapelfeldt, K. R., and Wyatt, M. C., "Target Selection for the LBTI Exozodi Key Science Program," ApJS 216, 24 (Feb. 2015).

[19] Bordé, P., Coudé du Foresto, V., Chagnon, G., and Perrin, G., "A catalogue of calibrator stars for long baseline stellar interferometry," A\&\&A 393, 183-193 (Oct. 2002).

[20] Mérand, A., Bordé, P., and Coudé du Foresto, V., "A catalog of bright calibrator stars for 200-m baseline near-infrared stellar interferometry," A\&\&A 433, 1155-1162 (Apr. 2005).

[21] Chelli, A., Duvert, G., Bourgès, L., Mella, G., Lafrasse, S., Bonneau, D., and Chesneau, O., "Pseudomagnitudes and differential surface brightness: Application to the apparent diameter of stars," A\&A 589, A112 (May 2016). 
[22] Gáspár, A., Rieke, G. H., and Balog, Z., "The Collisional Evolution of Debris Disks," ApJ 768, 25 (May 2013).

[23] Thureau, N. D., Greaves, J. S., Matthews, B. C., Kennedy, G., Phillips, N., Booth, M., Duchêne, G., Horner, J., Rodriguez, D. R., Sibthorpe, B., and Wyatt, M. C., "An unbiased study of debris discs around A-type stars with Herschel," MNRAS 445, 2558-2573 (Dec. 2014).

[24] Beichman, C. A., Bryden, G., Stapelfeldt, K. R., Gautier, T. N., Grogan, K., Shao, M., Velusamy, T., Lawler, S. M., Blaylock, M., Rieke, G. H., Lunine, J. I., Fischer, D. A., Marcy, G. W., Greaves, J. S., Wyatt, M. C., Holland, W. S., and Dent, W. R. F., "New Debris Disks around Nearby Main-Sequence Stars: Impact on the Direct Detection of Planets," ApJ 652, 1674-1693 (Dec. 2006).

[25] Su, K. Y. L., Rieke, G. H., Stansberry, J. A., Bryden, G., Stapelfeldt, K. R., Trilling, D. E., Muzerolle, J., Beichman, C. A., Moro-Martin, A., Hines, D. C., and Werner, M. W., "Debris Disk Evolution around A Stars," ApJ 653, 675-689 (Dec. 2006).

[26] Rieke, G. H., Su, K. Y. L., Stansberry, J. A., Trilling, D., Bryden, G., Muzerolle, J., White, B., Gorlova, N., Young, E. T., Beichman, C. A., Stapelfeldt, K. R., and Hines, D. C., "Decay of Planetary Debris Disks," ApJ 620, 1010-1026 (Feb. 2005).

[27] Eiroa, C., Marshall, J. P., Mora, A., Montesinos, B., Absil, O., Augereau, J. C., Bayo, A., Bryden, G., Danchi, W., del Burgo, C., Ertel, S., Fridlund, M., Heras, A. M., Krivov, A. V., Launhardt, R., Liseau, R., Löhne, T., Maldonado, J., Pilbratt, G. L., Roberge, A., Rodmann, J., Sanz-Forcada, J., Solano, E., Stapelfeldt, K., Thébault, P., Wolf, S., Ardila, D., Arévalo, M., Beichmann, C., Faramaz, V., GonzálezGarcía, B. M., Gutiérrez, R., Lebreton, J., Martínez-Arnáiz, R., Meeus, G., Montes, D., Olofsson, G., Su, K. Y. L., White, G. J., Barrado, D., Fukagawa, M., Grün, E., Kamp, I., Lorente, R., Morbidelli, A., Müller, S., Mutschke, H., Nakagawa, T., Ribas, I., and Walker, H., "DUst around NEarby Stars. The survey observational results," A\&SA 555, A11 (July 2013).

[28] Montesinos, B., Eiroa, C., Krivov, A. V., Marshall, J. P., Pilbratt, G. L., Liseau, R., Mora, A., Maldonado, J., Wolf, S., Ertel, S., Bayo, A., Augereau, J.-C., Heras, A. M., Fridlund, M., Danchi, W. C., Solano, E., Kirchschlager, F., del Burgo, C., and Montes, D., "Incidence of debris discs around FGK stars in the solar neighbourhood," A\&SA 593, A51 (Sept. 2016).

[29] Trilling, D. E., Bryden, G., Beichman, C. A., Rieke, G. H., Su, K. Y. L., Stansberry, J. A., Blaylock, M., Stapelfeldt, K. R., Beeman, J. W., and Haller, E. E., "Debris Disks around Sun-like Stars," ApJ 674, 1086-1105 (Feb. 2008).

[30] Greaves, J. S., Wyatt, M. C., Holland, W. S., and Dent, W. R. F., "The debris disc around $\tau$ Ceti: a massive analogue to the Kuiper Belt," MNRAS 351, L54-L58 (July 2004).

[31] Aumann, H. H., "IRAS observations of matter around nearby stars," PASP 97, 885-891 (Oct. 1985).

[32] Lawler, S. M., Beichman, C. A., Bryden, G., Ciardi, D. R., Tanner, A. M., Su, K. Y. L., Stapelfeldt, K. R., Lisse, C. M., and Harker, D. E., "Explorations Beyond the Snow Line: Spitzer/IRS Spectra of Debris Disks Around Solar-type Stars," ApJ 705, 89-111 (Nov. 2009).

[33] Koerner, D. W., Kim, S., Trilling, D. E., Larson, H., Cotera, A., Stapelfeldt, K. R., Wahhaj, Z., FajardoAcosta, S., Padgett, D., and Backman, D., "New Debris Disk Candidates Around 49 Nearby Stars," ApJ 710, L26-L29 (Feb. 2010).

[34] Aumann, H. H., "Spectral class distribution of circumstellar material in main-sequence stars," $A J$ 96, 1415-1419 (Oct. 1988).

[35] Kharchenko, N. V., Scholz, R., Piskunov, A. E., Roeser, S., and Schilbach, E., "2nd Cat. of Radial Velocities with Astrometric Data (Kharchenko+, 2007)," VizieR Online Data Catalog 3254, 0-+ (June 2007). 
[36] Gezari, D. Y., Schmitz, M., Pitts, P. S., and Mead, J. M., [Catalog of infrared observations, third edition] (June 1993).

[37] van Leeuwen, F., "Validation of the new Hipparcos reduction," A $\mathscr{J} A$ 474, 653-664 (Nov. 2007).

[38] Hoffmann, W. F., Hinz, P. M., Defrère, D., Leisenring, J. M., Skemer, A. J., Arbo, P. A., Montoya, M., and Mennesson, B., "Operation and performance of the mid-infrared camera, NOMIC, on the Large Binocular Telescope," in [Ground-based and Airborne Instrumentation for Astronomy V], Proc. SPIE 9147, 914710 (July 2014).

[39] Defrère, D., Hinz, P., Downey, E., Ashby, D., Bailey, V., Brusa, G., Christou, J., Danchi, W. C., Grenz, P., Hill, J. M., Hoffmann, W. F., Leisenring, J., Lozi, J., McMahon, T., Mennesson, B., Millan-Gabet, R., Montoya, M., Powell, K., Skemer, A., Vaitheeswaran, V., Vaz, A., and Veillet, C., "Co-phasing the Large Binocular Telescope: status and performance of LBTI/PHASECam," ArXiv e-prints (Jan. 2015).

[40] Mennesson, B., Serabyn, E., Hanot, C., Martin, S. R., Liewer, K., and Mawet, D., "New Constraints on Companions and Dust within a Few AU of Vega," ApJ 736, 14 (July 2011).

[41] Hanot, C., Mennesson, B., Martin, S., Liewer, K., Loya, F., Mawet, D., Riaud, P., Absil, O., and Serabyn, E., "Improving Interferometric Null Depth Measurements using Statistical Distributions: Theory and First Results with the Palomar Fiber Nuller," ApJ 729, 110 (Mar. 2011).

[42] Defrère, D., Hinz, P. M., Mennesson, B., Hoffmann, W. F., Millan-Gabet, R., Skemer, A. J., Bailey, V., Danchi, W. C., Downey, E. C., Durney, O., Grenz, P., Hill, J. M., McMahon, T. J., Montoya, M., Spalding, E., Vaz, A., Absil, O., Arbo, P., Bailey, H., Brusa, G., Bryden, G., Esposito, S., Gaspar, A., Haniff, C. A., Kennedy, G. M., Leisenring, J. M., Marion, L., Nowak, M., Pinna, E., Powell, K., Puglisi, A., Rieke, G., Roberge, A., Serabyn, E., Sosa, R., Stapeldfeldt, K., Su, K., Weinberger, A. J., and Wyatt, M. C., "Nulling Data Reduction and On-sky Performance of the Large Binocular Telescope Interferometer," ApJ 824, 66 (June 2016).

[43] Mennesson, B., Defrère, D., Nowak, M., Hinz, P., Millan-Gabet, R., Absil, O., Bailey, V., Bryden, G., Danchi, W., Kennedy, G. M., Marion, L., Roberge, A., Serabyn, E., Skemer, A. J., Stapelfeldt, K., Weinberger, A. J., and Wyatt, M., "Making high-accuracy null depth measurements for the LBTI exozodi survey," in [Optical and Infrared Interferometry and Imaging V], Proc. SPIE 9907, 99070X (Aug. 2016).

[44] Kelsall, T., Weiland, J. L., Franz, B. A., Reach, W. T., Arendt, R. G., Dwek, E., Freudenreich, H. T., Hauser, M. G., Moseley, S. H., Odegard, N. P., Silverberg, R. F., and Wright, E. L., "The COBE Diffuse Infrared Background Experiment Search for the Cosmic Infrared Background. II. Model of the Interplanetary Dust Cloud," ApJ 508, 44-73 (Nov. 1998).

[45] Kennedy, G. M., Wyatt, M. C., Bailey, V., Bryden, G., Danchi, W. C., Defrère, D., Haniff, C., Hinz, P. M., Lebreton, J., Mennesson, B., Millan-Gabet, R., Morales, F., Panić, O., Rieke, G. H., Roberge, A., Serabyn, E., Shannon, A., Skemer, A. J., Stapelfeldt, K. R., Su, K. Y. L., and Weinberger, A. J., "Exo-zodi Modeling for the Large Binocular Telescope Interferometer," ApJS 216, 23 (Feb. 2015).

[46] Lawler, S. M., Di Francesco, J., Kennedy, G. M., Sibthorpe, B., Booth, M., Vandenbussche, B., Matthews, B. C., Holland, W. S., Greaves, J., Wilner, D. J., Tuomi, M., Blommaert, J. A. D. L., de Vries, B. L., Dominik, C., Fridlund, M., Gear, W., Heras, A. M., Ivison, R., and Olofsson, G., "The debris disc of solar analogue $\tau$ Ceti: Herschel observations and dynamical simulations of the proposed multiplanet system," MNRAS 444, 2665-2675 (Nov. 2014).

[47] Feng, F., Tuomi, M., Jones, H. R. A., Barnes, J., Anglada-Escudé, G., Vogt, S. S., and Butler, R. P., "Color Difference Makes a Difference: Four Planet Candidates around $\tau$ Ceti," AJ 154, 135 (Oct. 2017). 
[48] Defrère, D., Absil, O., Berger, J.-P., Boulet, T., Danchi, W. C., Ertel, S., Gallenne, A., Hénault, F., Hinz, P., Huby, E., Ireland, M., Kraus, S., Labadie, L., Le Bouquin, J.-B., Martin, G., Matter, A., Mérand, A., Mennesson, B., Minardi, S., Monnier, J., Norris, B., Orban de Xivry, G., Pedretti, E., Pott, J.-U., Reggiani, M., Serabyn, E., Surdej, J., Tristram, K. R. W., and Woillez, J., "The path towards high-contrast imaging with the VLTI: the Hi-5 project," ArXiv e-prints (Jan. 2018).

[49] Defrère, D., Absil, O., Berger, J.-P., Boulet, T., Danchi, W. C., Ertel, S., Gallenne, A., Hénault, F., Hinz, P., Huby, E., Ireland, M., Kraus, S., Labadie, L., Le Bouquin, J.-B., Martin, G., Matter, A., Mérand, A., Mennesson, B., Minardi, S., Monnier, J., Norris, B., Orban de Xivry, G., Pedretti, E., Pott, J.-U., Reggiani, M., Serabyn, E., Surdej, J., Tristram, K. R. W., and Woillez, J., "Hi-5: a potential high-contrast thermal near-infrared imager for the VLTI," To appear in the same issue as the present paper. (2018).

[50] Nesvorný, D., Jenniskens, P., Levison, H. F., Bottke, W. F., Vokrouhlický, D., and Gounelle, M., "Cometary Origin of the Zodiacal Cloud and Carbonaceous Micrometeorites. Implications for Hot Debris Disks," ApJ 713, 816-836 (Apr. 2010).

[51] Marshall, J. P., Cotton, D. V., Bott, K., Ertel, S., Kennedy, G. M., Wyatt, M. C., del Burgo, C., Absil, O., Bailey, J., and Kedziora-Chudczer, L., "Polarization Measurements of Hot Dust Stars and the Local Interstellar Medium," ApJ 825, 124 (July 2016).

[52] Ertel, S., Wolf, S., Marshall, J. P., Eiroa, C., Augereau, J.-C., Krivov, A. V., Löhne, T., Absil, O., Ardila, D., Arévalo, M., Bayo, A., Bryden, G., del Burgo, C., Greaves, J., Kennedy, G., Lebreton, J., Liseau, R., Maldonado, J., Montesinos, B., Mora, A., Pilbratt, G. L., Sanz-Forcada, J., Stapelfeldt, K., and White, G. J., "A peculiar class of debris disks from Herschel/DUNES. A steep fall off in the far infrared," $A \mathscr{E} A \mathbf{5 4 1}$, A148 (May 2012).

[53] Ertel, S., Wolf, S., Metchev, S., Schneider, G., Carpenter, J. M., Meyer, M. R., Hillenbrand, L. A., and Silverstone, M. D., "Multi-wavelength modeling of the spatially resolved debris disk of HD 107146," A\& A 533, A132+ (Sept. 2011).

[54] Ertel, S., Marshall, J. P., Augereau, J.-C., Krivov, A. V., Löhne, T., Eiroa, C., Mora, A., del Burgo, C., Montesinos, B., Bryden, G., Danchi, W., Kirchschlager, F., Liseau, R., Maldonado, J., Pilbratt, G. L., Schüppler, C., Thébault, P., White, G. J., and Wolf, S., "Potential multi-component structure of the debris disk around HIP 17439 revealed by Herschel/DUNES," A\&A 561, A114 (Jan. 2014).

[55] Spalding, E., Hinz, P., Ertel, S., Maier, E., and Stone, J., "Towards controlled Fizeau observations with the Large Binocular Telescope," To appear in the same issue as the present paper. (2018).

[56] Wenger, M., Ochsenbein, F., Egret, D., Dubois, P., Bonnarel, F., Borde, S., Genova, F., Jasniewicz, G., Laloë, S., Lesteven, S., and Monier, R., "The SIMBAD astronomical database. The CDS reference database for astronomical objects," A\&BS 143, 9-22 (Apr. 2000).

[57] Ochsenbein, F., Bauer, P., and Marcout, J., "The VizieR database of astronomical catalogues," A $\mathscr{E} A S \mathbf{1 4 3}$, 23-32 (Apr. 2000).

[58] Hunter, J. D., "Matplotlib: A 2d graphics environment," Computing In Science $\mathscr{G}$ Engineering 9(3), 90-95 (2007).

[59] Astropy Collaboration, Robitaille, T. P., Tollerud, E. J., Greenfield, P., Droettboom, M., Bray, E., Aldcroft, T., Davis, M., Ginsburg, A., Price-Whelan, A. M., Kerzendorf, W. E., Conley, A., Crighton, N., Barbary, K., Muna, D., Ferguson, H., Grollier, F., Parikh, M. M., Nair, P. H., Unther, H. M., Deil, C., Woillez, J., Conseil, S., Kramer, R., Turner, J. E. H., Singer, L., Fox, R., Weaver, B. A., Zabalza, V., Edwards, Z. I., Azalee Bostroem, K., Burke, D. J., Casey, A. R., Crawford, S. M., Dencheva, N., Ely, J., Jenness, T., Labrie, K., Lim, P. L., Pierfederici, F., Pontzen, A., Ptak, A., Refsdal, B., Servillat, M., and Streicher, O., "Astropy: A community Python package for astronomy," A $\xi A$ 558, A33 (Oct. 2013). 